\title{
Negative data in DEA: a directional distance approach applied to bank branches
}

\author{
MCA Silva Portela ${ }^{1,2 *}$, E Thanassoulis ${ }^{2}$ and G Simpson ${ }^{2}$ \\ ${ }^{1}$ Universidade Católica Portuguesa, Centro Regional do Porto, Porto, Portugal; and ${ }^{2}$ Aston Business School, \\ Birmingham, UK
}

\begin{abstract}
This paper is drawn from the use of data envelopment analysis (DEA) in helping a Portuguese bank to manage the performance of its branches. The bank wanted to set targets for the branches on such variables as growth in number of clients, growth in funds deposited and so on. Such variables can take positive and negative values but apart from some exceptions, traditional DEA models have hitherto been restricted to non-negative data. We report on the development of a model to handle unrestricted data in a DEA framework and illustrate the use of this model on data from the bank concerned.
\end{abstract}

Keywords: bank branches; data envelopment analysis; negative data in DEA

\section{Introduction}

Data envelopment analysis (DEA) as first introduced by Farrell ${ }^{1}$ and later developed by Charnes $e t a l^{2}$ is an efficiency assessment tool that implicitly assumes non-negativity of all inputs and outputs. This assumption is not, however, always satisfied as was the case in our application to bank branches, which led to the development of alternative models aiming at assessing efficiency in the presence of negative data.

Bank branches have been facing major challenges over the last years relating mainly to the development of alternative distribution channels competing with bank branches. The main implication of this was a change in the role played by bank branches that went from a transaction- to a sales-based role (see eg Drake and Howeroft ${ }^{3}$ ). This changing role of bank branches has been mainly acknowledged in the marketing literature (see eg Howcroft and Beckett, ${ }^{4}$ Howcroft $^{5}$ and Howland ${ }^{6}$ ), but only recently it has been recognized in the efficiency measurement literature. For example, Cook et $a l^{7}$ and Cook and Hababou ${ }^{8}$ divide the activities of bank branches into two sets: sales and service, and propose models to assess efficiency in each case. We too distinguish between sales and service in this paper, but we follow a different approach to measuring operational efficiency. Increases in sales and customers are seen as the main objectives of our bank branches. Growth in these variables (ie positive outputs) is obviously better than decline (negative outputs), but a negative output is not bad in itself. In fact, a bank branch may consciously choose to focus

*Correspondence: MCA Silva Portela, Universidade Católica Portuguesa, Centro Regional do Porto, R. Diogo Botelho, 1327 Porto 4169-005, Portugal.

E-mail: csilva@porto.ucp.pt more on one of the outputs (say customer base growth) at the expense of focusing less on the other (say sales (credit advances) growth). These two strategies are equally valid although they may mean neglecting one output in favour of another. The neglected output may thus suffer a decrease, and growth will be negative.

Traditionally, negative data are handled in efficiency applications through some data transformation (eg adding an arbitrary large number to all values of a given variable) so that all negative data are turned into positive data (see eg Pastor ${ }^{9}$ and Lovell $^{10}$ ). Such transformation of the data may have implications for the solution, classification, or ordering of the DEA results. ${ }^{11}$ There are, however, some models whose solution is invariant to data transformations, which are usually referred to as translation invariant. In the presence of negative data, the most often used model is the variable returns to scale (VRS) additive model of Charnes et $a l,{ }^{12}$ which is translation invariant as demonstrated by Ali and Seiford. ${ }^{13}$ The additive model is not, however, in its original form, units invariant (independent of scale of measurement of the variables). Owing to this limitation, Lovell and Pastor ${ }^{14}$ put forward a unit-invariant version of the additive model that uses a weighted sum of slacks where the weights are the inverse of the standard deviations of the corresponding input and output variables (see also Pastor ${ }^{15}$ and Thrall ${ }^{16}$ ). The main advantage of the additive model is that it can be applied to negative data directly without any need to transform them subjectively. However, the additive model has some drawbacks, namely the fact that it yields in respect of an inefficient unit the 'furthest' targets on the production frontier, while at the same time it does not yield an efficiency measure that can be readily interpreted. Thus, 
the model does not yield very practical guidance as to how a unit might improve its performance nor does it make it possible to readily rank units on performance. The VRS model of Banker et al $^{17}$ (also known as BCC model) is able to provide an efficiency score in the presence of negative data, but this cannot be achieved without transforming the data. In addition, the BCC model has restricted translation invariance (it is translation invariant on inputs if it is output oriented, and translation invariant on outputs if it is input oriented, ${ }^{14,15}$ meaning that the efficiency scores may depend on the way data are translated.

Thus, there is no DEA model to date that can be used with negative data directly without any need to transform them, while at the same time it yields an efficiency score that can be readily used to compare units. In this paper, we propose DEA models that provide efficiency scores, similar in meaning to radial efficiencies traditionally used in DEA, while at the same time negative data can be used without the need to transform them subjectively. This is an important advantage over existing approaches to deal with negative data.

Our approach is inspired by the well-known directional distance model of Chambers et $a l^{18,19}$ and it provides efficiency scores that can be directly used to rank and compare production units when some inputs and/or outputs are negative. Targets resulting from our procedure, and from a variant of this procedure, are also analysed in this paper and it is shown that our models, in general, provide closer targets than existing models in the literature. Closer targets represent a useful practical feature because they would prove easier for the unit to attain and have been explored for the case when all data are positive in a number of papers (eg Coelli, ${ }^{20}$ Frei and Harker, ${ }^{21}$ Cherchye and Van Puyenbroeck, ${ }^{22}$ Portela et $a l^{23}$ among others).

The paper is structured as follows. Some issues arising in the context of negative data will be explored in the next section. Then, we present a directional distance procedure for dealing with negative data in DEA, and after that issues relating with the closeness of the targets obtained from our approach and also other well-known models in the literature are addressed. In the penultimate section, our procedure is applied to measure the operational efficiency of a set of Portuguese bank branches, and finally the paper concludes.

\section{Negative data: implications in DEA}

Negative data may arise in DEA due to the use of inputoutput variables that relate to changes in their values like changes in clients or accounts from one period to the other, ${ }^{9}$ or due to the use of variables like profit that may take both positive and negative values. ${ }^{24}$ Negative inputs or outputs may also arise artificially as a way to deal with undesirable inputs or outputs. ${ }^{11}$
We can readily demonstrate that an assumption of CRS is not possible in technologies where negative data can exist. A CRS technology assumes that any activity can be 'radially expanded or contracted to form other feasible activities' (Färe et $a l,{ }^{25} \mathrm{p} \mathrm{50)}$. Take a set of only two units, A and B, represented by activity vectors $\left(x, y_{1}, y_{2}\right)$, where $x$ is input and $y_{1}$ and $y_{2}$ outputs. Assuming that output 1 is negative, consider that $\mathrm{A}$ and $\mathrm{B}$ equal $(1,-1,1)$ and $(1,-2,3)$, respectively. Unit $\mathrm{A}$ has higher productivity in $y_{1}$ and $\mathrm{B}$ has higher productivity in $y_{2}$, and therefore both units are CRS efficient (see Chen and $\mathrm{Ali}^{26}$ ). However, the additive CRS model shows only unit B efficient. In fact, it is possible to contract unit $\mathrm{B}$ (say by $50 \%$ ) radially and find a feasible point (under the CRS assumption) dominating unit A (eg $0.5 \mathrm{~B}=(0.5,-1,1.5)$ dominates $\mathrm{A})$. The productivity ratios, however, remain unchanged and as such this result is clearly wrong. The assumption behind CRS DEA models that any proportion of an efficient unit is also efficient is therefore only valid for non-negative data.

In the presence of negative data VRS technologies need to be assumed. However, the use of radial measures of efficiency traditionally used in VRS DEA models is problematic. To illustrate the point consider the example in Figure 1, where two outputs are represented (output 2 is positive and output 1 may be negative) and all units have the same input. Assessing the efficiency of unit U3 using, for example, the radial output-oriented $\mathrm{BCC}$ model ${ }^{17}$ without transforming the data, implies an expansion of both outputs by a multiple greater than 1. This, however, implies a movement of the inefficient unit U3 to the frontier in the direction shown by the arrow in Figure 1. This movement is not desired since the negative output is being expanded making it oven worse. Clearly, positive radial expansion factors applied to negative data lead in the opposite direction to the one we would wish to follow to improve performance. The addition of a constant to the negative output (output 1 in Figure 1) would move the frontier to the positive quadrant and the right direction would be followed by $\mathrm{U} 3$ towards the frontier. The output efficiency score would, however, depend on the value of the constant added to the negative output

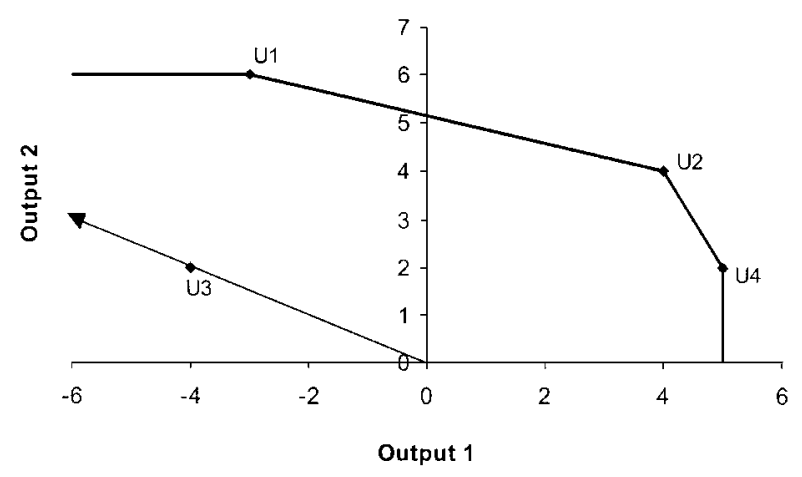

Figure 1 Example with one negative output. 
vector, and the resulting radial efficiency score would be hard to interpret in the light of the negative data it, in fact, represents.

Note that the treatment of negative data is in a way similar to the treatment of undesirable inputs and/or outputs, since both negative data and undesirable outputs need to be constrained to move in a direction that is contrary to the direction used in traditional DEA models. Several approaches exist to deal with undesirable outputs as can be seen in the recent review of Allen ${ }^{27}$ and Dyckhoff and Allen. ${ }^{28}$ One of these approaches is based on the directional distance function, and was first proposed by Chung et al. ${ }^{29}$ In this paper, we use a related approach, also based on the directional distance model, to deal with negative data.

\section{A directional distance function approach to coping with negative data in DEA}

Consider a set of units $J=\{1, \ldots, n\}$, with input levels $x_{i j}$, $i=1, \ldots, m$ and output levels $y_{r j}, r=1, \ldots, s$, and unit $o \in J$ which is to be assessed. The generic directional distance model as proposed by Chambers et al ${ }^{18,19}$ is in (1) for the case of VRS and with input and output vectors in $R^{m+s}$.

$$
\begin{aligned}
& \max \left\{\beta_{o} \mid \sum_{j=1}^{n} \lambda_{j} y_{r j} \geqslant y_{r o}+\beta_{o} g_{y_{r}}, r=1, \ldots, s,\right. \\
& \sum_{j=1}^{n} \lambda_{j} x_{i j} \leqslant x_{i o}-\beta_{o} g_{x_{i}}, \quad i=1, \ldots, m, \\
& \left.\sum_{j=1}^{n} \lambda_{j}=1, \lambda_{j}, \beta_{o}, g_{x_{i}}, g_{y_{r}} \geqslant 0\right\}
\end{aligned}
$$

Model (1) is defined in the most general non-oriented case as it looks simultaneously for input contraction and output expansion. Oriented models can be derived from (1) by setting respectively $g_{y_{r}}$ or $g_{x_{i}}$ equal to 0 . When data are strictly positive a usual choice for the directional vectors $\left(g_{x_{i}}, g_{y_{r}}\right)$ are the observed input and output levels. When some data are negative, the use of observed input and output levels would violate the last constraint of model (1), which is intended to ensure that inputs and outputs do not worsen from their observed levels in the solution the model yields.

We modify the model in (1) to ensure that it yields improving solutions even when some of the data are negative. Specifically, and for a given data set, consider an ideal point defined as $I=\left(\max _{J} \mathbf{y}_{\mathbf{j}}, \quad r=1, \ldots, s, \min _{J} \mathbf{x}_{\mathbf{j}}\right.$, $i=1, \ldots, m)$. We can now define the vectors $R_{r o}$ and $R_{i o}$ in (2), to which we refer as the range of possible improvement of unit $o$.

$$
\begin{aligned}
R_{r o}= & \max _{J}\left\{y_{r j}\right\}-y_{r o}, \quad r=1, \ldots, s \\
& \text { and } R_{i o}=x_{i o}-\min _{J}\left\{x_{i j}\right\}, \quad i=1, \ldots, m
\end{aligned}
$$

Although there is no evidence that any unit can actually exist at the ideal point $I$ the range of possible improvement in (2) can be seen as a surrogate for the maximum improvement that unit $o$ could, in principle, achieve on each input and output. Such an improvement can never be negative, and therefore the range vectors in (2) satisfy the non-negativity restrictions on the direction vectors used in (1). Under VRS units that have the maximum value on some output or the minimum value on some input are always $100 \%$ efficient. ${ }^{26}$ Thus, the range of possible improvement we use is determined by the efficient units' input/output levels, which is already a characteristic inherent in the classical DEA model (eg Thanassoulis, ${ }^{30}$ Chapter 3).

Note that this contrasts with other notions of 'range' used in the literature such as by Cooper et al,${ }^{31}$ where range of a variable is defined as its maximum observed minus its minimum observed value. In such a range, worst performance as given by maximum inputs and minimum outputs affects the results of the model. This is because worst performance is included in the definition of the range and efficiency results depending on the range defined. Another notion of 'range' related with that defined in (2) has been introduced by Bogetoft and Hougaard ${ }^{32}$ and also used by Asmild et al. ${ }^{33}$ Bogetoft and Hougaard ${ }^{32}$ introduce a 'potential improvements approach' using the input-oriented directional distance function, where the directional input vector is the difference between the observed input and an ideal reference input. This ideal input vector, however, is specific to each production unit reflecting the 'largest possible reduction in each input with all other inputs kept fixed' (Bogetoft and Hougaard, ${ }^{32}$ p 235). To the authors' knowledge, the use of the range direction as specified in (2) has never been used before in the literature.

\section{Range directional model}

Based on the notion of the range of possible improvement in (2), we define the range directional model (RDM) as shown in (3).

$$
\begin{aligned}
& \max \left\{\beta_{o} \mid \sum_{j=1}^{n} \lambda_{j} y_{r j} \geqslant y_{r o}+\beta_{o} R_{r o}, \quad r=1, \ldots, s,\right. \\
& \sum_{j=1}^{n} \lambda_{j} x_{i j} \leqslant x_{i o}-\beta_{o} R_{i o}, \quad i=1, \ldots, m, \\
& \left.\sum_{j=1}^{n} \lambda_{j}=1, \lambda_{j} \geqslant 0\right\}
\end{aligned}
$$

The RDM in (3) is translation invariant and units invariant, two important characteristics in DEA models that can deal with negative data.

To prove translation invariance, assume that an amount $K_{r}$ is added to each output and $V_{i}$ to each input. 
The constraints in (3), therefore, become $\sum_{j=1}^{n} \lambda_{j}\left(y_{r j}+K_{r}\right) \geqslant$ $\left(y_{r o}+K_{r}\right)+\beta_{o} R_{r o}$ and $\sum_{j=1}^{n} \lambda_{j}\left(x_{i j}+V_{i}\right) \leqslant\left(x_{i o}+V_{i}\right)-\beta_{o} R_{i o}$. Note that the range of improvement does not change with the addition of a constant to each input and output. The lefthand side of the output inequality $\left(\sum_{j=1}^{n} \lambda_{j}\left(y_{r j}+K_{r}\right)\right)$ is equivalent to $\sum_{j=1}^{n} \lambda_{j} y_{r j}+K_{r} \sum_{j=1}^{n} \lambda_{j}$. As $\sum_{j=1}^{n} \lambda_{j}=1$, then the constraints changed with $K_{r}$ reduce to the constraints in model (3). The same happens with the input constraints changed by $V_{i}$. Note that VRS has been assumed in RDM. It is only under VRS that we can have translation invariance.

To prove units invariance assume that all levels of input $i$ are multiplied by $\alpha_{i}$, and of output $r$ by $\gamma_{r}$. This results in the following modified constraints of (3): $\sum_{j=1}^{n} \lambda_{j} \gamma_{r} y_{r j} \geqslant$ $\gamma_{r} y_{r o}+\beta_{o} \gamma_{r} R_{r o}$ and $\sum_{j=1}^{n} \lambda_{j} \alpha_{i} x_{i j} \leqslant \alpha_{i} x_{i o}-\beta_{o} \alpha_{i} R_{i o}$. These constraints reduce to those in (3), whose solution, therefore, does not change when the unit of measurement changes.

The range of improvement $R_{r o}$ or $R_{i o}$ may be zero for some output or input. This is in line with intuition, because a range of zero improvement means that the unit has achieved on that variable a large enough value (for outputs) so that we have no observed evidence how that value might rise even further by, for example, compensating input rises or reductions in other output values. An analogous reasoning can be advanced as to why zero ranges for minimum observed input values are intuitive. Note that a constraint associated with a zero range is necessarily binding (target values equal observed values).

\section{Interpreting $\beta$ in model RDM}

At the optimal solution to model RDM at least one constraint is binding, meaning that $\beta$ equals $\left(y_{r}^{*}-y_{r o}\right) / R_{r o}$ or $\left(x_{i o}-x_{i}^{*}\right) / R_{i o}$ for at least an output $r$ or an input $i$. The star stands for the target value obtained at the optimal solution to model (3). This means $\beta$ is equal to the ratio of an optimal slack (that projects unit $o$ on the frontier) to the maximum possible slack (given by the range) unit $o$ had on that variable. Seen in this way $\beta$ is clearly an inefficiency measure. The RDM efficiency measure, $1-\beta$, is therefore defined as $\left(\operatorname{Max}_{J}\left\{y_{r j}\right\}-y_{r}^{*}\right) /\left(\operatorname{Max}_{J}\left\{y_{r j}\right\}-y_{r o}\right)$ if a binding constraint corresponds to output $r$, or $\left(x_{i}^{*}-\operatorname{Min}_{J}\left\{x_{i j}\right\}\right) /\left(x_{i o}-\operatorname{Min}_{J}\left\{x_{i j}\right\}\right)$ if a binding constraint corresponds to input $i$. As target outputs (target inputs) cannot be lower (higher) than observed outputs (observed inputs), the numerator of $1-\beta$ is never larger than the denominator, meaning that the upper bound of $1-\beta$ is 1 . Efficiency of 1 will only be achieved when the observed are also the target values for the inputs and outputs of unit $o$ as is the case in traditional DEA models. $^{2}$

The RDM efficiency measure can be better interpreted with the aid of Figure 2 (depicting the same units as Figure 1), where we are assuming an output-oriented RDM model.

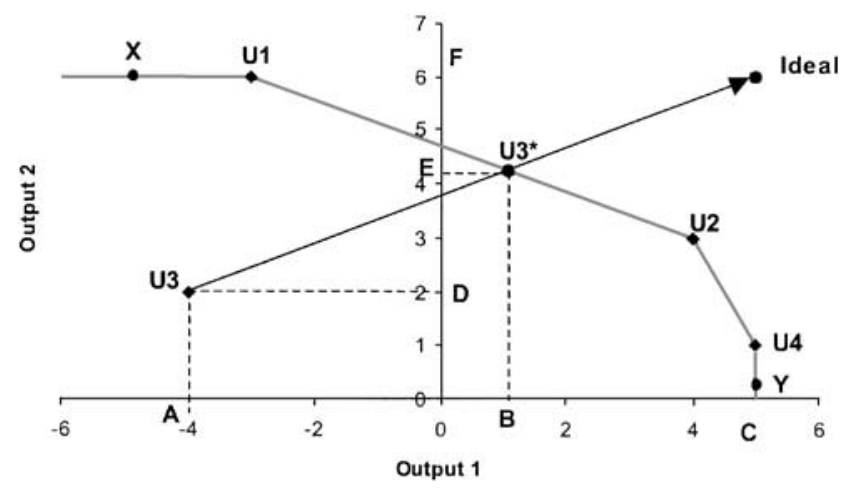

Figure 2 RDM in a two-output example.

The efficiency measure $1-\beta$ of $\mathrm{U} 3$ equals the ratio $\overline{C B} / \overline{C A}$, which in turn equals the ratio $\overline{F E} / \overline{F D}$. Note that $\overline{C B} / \overline{C A}$ measures the distance between the level of output 1 at the observed point $\mathrm{U} 3$ and its target point $\mathrm{U} 3^{*} . \overline{F E} / \overline{F D}$ is interpreted in a similar manner in respect to the level of output 2. Thus, we have for U3 a value of $1-\beta$ equal to $(5-1.07273) /(5-(-4))=(6-4.25455) /(6-2)=43.36 \%$, reflecting the relative distance between $\mathrm{U} 3$ and its target $\mathrm{U} 3{ }^{*}$.

Note that there is close similarity between the RDM efficiency measure and radial measures of efficiency traditionally used in DEA. The difference is in the reference point used to measure efficiency. In the RDM case, the reference point is not the origin used in traditional DEA models but rather the ideal point we defined using (2). In fact, if we rotate Figure 2 suitably we can arrive at Figure 3 in which the ideal point occupies the position of the origin in traditional DEA models.

Using Figure 3, it is easy to see that the efficiency measure yielded by model RDM, $1-\beta$, is a distance measure between the observed and its target point with reference to the ideal point. The lower this distance the higher the value of $1-\beta$ and the more efficient a unit will be. To see this note that the direction of improvement followed by inefficient units U3 and $\mathrm{U} 5$ in Figure 3 is defined with reference to the ideal point, a role played by the origin in traditional DEA models. Our efficiency measure has the same geometric interpretation as radial measures in DEA provided the ideal point is treated as the origin. Consider for example U3 and define two vectors $\vec{A}=\overrightarrow{I-T}$ that goes from ideal (I) to target (T) point, and $\vec{B}=\overrightarrow{I-O}$ that goes from ideal to observed $(O)$ point. Then the efficiency measure $(1-\beta)$ of $\mathrm{U} 3$ is given by the ratio between the length of these two vectors, that is by $\|A\| /\|B\|=\|I-T|| /|| I-O\|$, exactly as would be under traditional DEA, had the point $I$ been the origin. (For example, assuming an input-oriented DEA model, where data are assumed to be non-negative, consider the ratio $\|T\| /$ $\|O\|$ where $(T)$ is an input target vector and $(O)$ is an input observed vector. $T$ being the radial input target vector it equals $\theta O$, where $\theta$ is the input efficiency score, meaning that the above ratio of norms reduces to $\theta$.) 


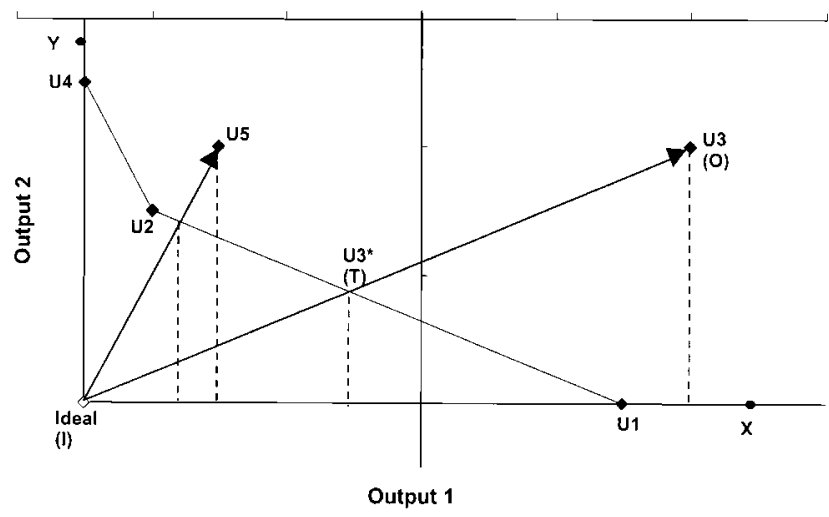

Figure 3 Figure 2 after rotation.

\section{Pareto-efficiency}

Efficient units will necessarily have in the RDM model an optimal $1-\beta$ equal to 1 , but this is not a sufficient condition for Pareto-efficiency. The Pareto-optimality conditions for model RDM are, therefore, two: (i) $\beta_{o}=0$, and (ii) all constraints of (3) are satisfied in equality (ie all slacks are 0 ). Note that although the RDM model does not assure projection on Pareto-efficient targets, it may in some cases correctly identify weak efficiency. This is the case for units $X$ and $Y$ in Figure 2 (or in Figure 3), whose assessment through the RDM model yields a value of $1-\beta$ different from 1 . This is an interesting characteristic of the RDM model that, although behaving as a radial model, can in some cases identify weak efficient units. Units located on an inefficient part of the frontier have at least one of the ranges equal to 0 , which has no influence over the value of $\beta$. As $\beta$ is maximized in model RDM (see (3)) any inefficiencies in the factors that have non-zero range may be found because these will push $\beta$ to be greater than 0 .

Note that to find Pareto-efficient targets one can solve (3) in a first stage and the additive model in a second stage as described in Ali and Seiford. ${ }^{34}$ Alternatively, Pareto-efficient solutions can be found by solving the additive model in a first stage to identify those units that are Pareto-efficient and then restrict the reference set in the RDM model to those Pareto-efficient units. In our empirical implementation detailed in the next section, we have chosen the latter approach.

The aforementioned implies that the efficiency score $1-\beta$ is not able to incorporate all the sources of inefficiency, since slack values are not reflected in the value of $\beta$. Ranking units based on an efficiency measure that does not include all the sources of inefficiency may result in a biased ranking especially if slacks are high. We can, however, use the ratio of the norms $(\|A\| /|| B\|=|| I-T|| /|| I-O\|$, as defined previously) to account for all inefficiencies including those from slacks as long as target levels considered in the computation of $A$ and $B$ are Pareto-efficient (see eg Cherchye and Van
Puyenbroeck $\left.{ }^{35,36}\right)$. Note, however, that when Pareto-efficient targets are used in $A$ and $B$ these two vectors are not necessarily collinear, meaning that the resulting efficiency measure is dependent on units of measurement. In order to avoid this problem, the ratio of norms $(\|A\| /|| B||)$ should be used on normalized data only.

\section{Target setting under negative data in DEA}

In the RDM model, the direction towards the production frontier is in a sense 'biased' towards the factors with the largest potential for improvement. That is, the model seeks targets such that the factors on which the unit has the largest difference from the 'best' values observed elsewhere are those where improvement is given priority. Thus, in a sense the model seeks targets so that the unit will improve in those factors where it does 'worse' relative to other units, and therefore the targets may prove hard for the unit to achieve in the short run.

This section puts forth an alternative direction of improvement of inputs and outputs so that the unit will identify targets where the factors on which it does best are given priority to improve. Such targets will normally prove easier for the unit to attain in the short term. This direction uses the inverse of the ranges in (2) in the context of model (3). The resulting model, referred to as Inverse RDM (IRDM), is

$$
\begin{aligned}
& \max \left\{\beta_{o} \mid \sum_{j=1}^{n} \lambda_{j} y_{r j} \geqslant y_{r o}+\beta_{o} \frac{1}{R_{r o}}, r=1, \ldots, s,\right. \\
& \sum_{j=1}^{n} \lambda_{j} x_{i j} \leqslant x_{i o}-\beta_{o} \frac{1}{R_{i o}}, i=1, \ldots, m, \\
& \left.\sum_{j=1}^{n} \lambda_{j}=1, \lambda_{j} \geqslant 0\right\}
\end{aligned}
$$

For ranges in (4) which are 0 , division by 0 is avoided and we use 0 as the coefficient of the corresponding $1 / R_{\text {io }}$ or $1 / R_{r o}$. This treatment of zero ranges ensures that the corresponding input or output has within the targets derived the same value as that observed at the unit concerned. This matches the treatment of zero ranges in the RDM model.

Model (4) is translation invariant (the proof for translation invariance of (4) is exactly the same as for model (3), because the range for improvement does not change when the same constant is added to inputs and/or outputs), but it is not units invariant. Assuming, for example, that all levels of output $r$ are multiplied by $\gamma_{r}$, we have $\sum_{j=1}^{n} \lambda_{j} \gamma_{r} y_{r j} \geqslant \gamma_{r} y_{r o}+\beta_{o}\left(1 / \gamma R_{r o}\right)$, which is not equivalent to the constraint for output $r$ in (4). In order to circumvent this problem, we can use normalized data in model (4), so that its solution is not dependent on unit of measurement. Using 
normalized data, and ranges calculated on this normalized data, makes model (4) units invariant. (As $\sum_{j=1}^{n} \lambda_{j}\left(y_{r j}\right)$ $\left.Y_{r}\right) \geqslant y_{r o} / Y_{r}+\beta\left(Y_{r} / R_{r o}\right)$ (where $Y_{r}$ is the maximum output $r$ ), is equal to $\sum_{j=1}^{n} \lambda_{j}\left(\alpha y_{r j} / \alpha Y_{r}\right) \geqslant\left(\alpha y_{r o} / \alpha Y_{r}\right)+\beta\left(\alpha Y_{r} / \alpha R_{r o}\right)$. Note that the same is valid for input constraints.) The IRDM model is, therefore, translation and units invariant on normalized data. Model (4) is applied in this paper only to data that have been previously normalized by a non-negative value.

Model (4) shall be used in this paper for target setting purposes only. This is because efficiency measure it yields does not have a straightforward interpretation as will be seen next.

The IRDM efficiency score $1-\beta$ in (4) measures the distance from an observed point to a target point with reference to some ideal point. However, the IRDM model works as if a different ideal point was defined for each unit, which represents a problem in interpreting and comparing efficiency scores accruing from this model.

To illustrate this consider the optimum $\beta$, as resulting from (4), after normalising outputs by $Y_{r}$ and inputs by $X_{i}$, which is $\left.\left(y_{r}^{*}-y_{r o}\right) / Y_{r}\right) /\left(Y_{r} / R_{r o}\right)$ or $\left(\left(x_{i o}-x_{i}^{*}\right) / X_{i}\right) /\left(X_{i} / R_{i o}\right)$ when respectively the constraint relating to output $r$ or input $i$ is binding at the optimal solution to the IRDM model. Consider now a new range of improvement as given by $R_{r o}^{\prime}=Y_{r} / R_{r o}$ for outputs, and $R_{i o}^{\prime}=X_{i} / R_{i o}$ for inputs, and normalised target and observed levels equal to $y_{e}{ }^{*}=y_{e}{ }^{*} / y_{e}$, $y_{e o}^{\prime}=y_{e o} / y_{e}, x_{i}^{\prime *}=x_{i}^{*} / X_{i}$, and $x_{i o}^{\prime}=x_{i o} / X_{i}$. The optimal value of $\beta$ in the IRDM model reduces therefore to $\left.\left(y_{r}{ }^{\prime *}-y_{r o}^{\prime}\right) / R_{r o}^{\prime}\right)$ or $\left(x_{i o}^{\prime}-x_{i}^{\prime *}\right) / R_{i o}^{\prime}$ when respectively the constraint relating to output $r$ or input $i$ is binding at the optimal solution to the IRDM model. The above IRDM efficiency relates directly with the RDM efficiency measure, where the range of possible improvement is defined in relation to an ideal point $I^{\prime}=\left(y_{r o}^{\prime}+R_{r o}^{\prime}, x_{i o}^{\prime}-R_{i o}^{\prime}\right)$. Point $I^{\prime}$ is no longer fixed as it was in the case of the RDM model, but varies for each production unit.

Since the IRDM model works as if different ideal points were defined for each production unit under assessment, the IRDM efficiency measures are not comparable within themselves nor with RDM efficiency scores. This means that the IRDM model should not be used to rank and compare units but just for target setting purposes.

\section{Closest targets and the RDM models}

The IRDM model gives priority to improve the factors on which production units perform best. As a result one expects targets derived from this model to be less demanding (closer) than those resulting from the RDM model. The IRDM model may therefore be a good alternative to more complicated procedures of finding closest targets to inefficient units. For example, the procedure developed by Charnes $e t a l^{37}$ for calculating the radius of stability (minimum change needed to change the classification of a unit) can be used for calculating targets with the minimum $L_{1}$ distance. This procedure is not, however, units invariant and implies solving several linear programming models $(m+s)$, each being an additive model that maximizes slack variables in turn (see also Briec, ${ }^{38}$ who puts forward the same model for finding the minimum $L_{1}$ projection). Portela et $a l^{23}$ have also developed a procedure for finding closest targets to inefficient units. Their procedure is, however, based on a measure that cannot be directly applied in the presence of negative data as it is based on ratios of target to observed input or output levels (which would be meaningless in case observed data are negative).

The IRDM model does not assure closeness on any criteria (such as any $L_{p}$ metric) but by focusing improvements on the factors at which the unit is already good at it provides, in principle, targets that are near the closest.

We illustrate this point through the example that has been used previously (see eg Figure 2), namely showing distances from unit U3 to alternative targets. We consider six different targets to unit $\mathrm{U} 3$ in Table 1: target $\mathrm{U} 1(-3,6)$ is the closest target to this unit according to the procedure of Charnes et $a l{ }^{37}$ target $\mathrm{U} 2(4,3)$ results from solving the translation invariant additive model of Lovell and Pastor; ${ }^{14}$ target $\mathrm{BCC} 1(-2.1765,5.65)$ results from solving the BCC model, where output 1 is transformed into a positive output by adding 5 ; target $\mathrm{BCC} 2(1.8125,3.9375)$ results from solving the BCC model, where output 1 is transformed into a positive output by adding 10; target RDM (1.073, 4.255) results from the RDM model; and target IRDM $(-2.793$, 5.911) results from the IRDM model. Note that the target levels for output 1 obtained directly from the BCC model are always non-negative due to the transformation imposed, but we then re-transformed output 1 targets by subtracting a value of 5 and 10 , respectively, for BCC1 and BCC 2 .

U1 is the target yielding the smallest $L_{1}$ norm, but this is not true for the other $L_{p}$ metrics, where the IRDM target performs better than the procedure of Charnes et al. ${ }^{37}$ Note that the $\mathrm{BCC} 1$ target performs very well in most $L_{p}$ norms except in the $L_{1}$. Note also that the translation of the data has a big impact on the target levels obtained and also on their distance from observed levels.

Results in terms of $L_{p}$ metrics should, however, be interpreted carefully because these metrics are units depen-

Table 1 Distance of U3 from some targets

\begin{tabular}{llclc}
\hline Target & $L_{1}$ & $L_{2}$ & $L_{\infty}$ & $\|A\| /\|B\|(\%)$ \\
\hline Unit U1 & 5 & $\sqrt{17}$ & 4 & 83.35 \\
Unit U2 & 9 & $\sqrt{65}$ & 8 & 28.05 \\
BCC1 & 5.47 & $\sqrt{16.63}$ & 3.65 & 74.84 \\
BCC2 & 7.75 & $\sqrt{37.54}$ & 5.81 & 37.73 \\
RDM Tgt & 7.33 & $\sqrt{30.82}$ & 5.07 & 43.64 \\
IRDM Tgt & 5.12 & $\sqrt{16.75}$ & 3.9 & 81.20 \\
\hline
\end{tabular}


dent. This means that they are only valid when variables are measured on the same scale or else they can induce completely wrong interpretations. The units independent ratio of norms $(\|A\| /|| B||=|| I-T|| /|| I-O \|)$ can be used to calculate the distance between any observed vector and a target vector with reference to the ideal point. For the alternative targets shown in Table 1, we calculated the ratio of norms based on values normalized by the maximum and used a common ideal point as defined by maximum outputs $[I=(5,6)]$. This means that all the ratios of norms in Table 1 are comparable among themselves. The highest value for this ratio happens for projection of $\mathrm{U} 3$ on $\mathrm{U} 1$, with the second best being attributed to the IRDM target. Note that the additive target (U2) shows the highest distances from targets in all the criteria, and the RDM target lies somewhere between the IRDM and the additive model's targets.

The above results are illustrative, but they support our argument that the IRDM model has the advantage of looking for closer projections on the efficient frontier when compared to the RDM or to the additive model. The empirical application presented on the next section will further deal with the issue of the closest targets.

\section{An application of the RDM to bank branches}

In this section, we describe how we have used the RDM model developed in this paper to estimate targets and to measure the operational efficiency of a set of Portuguese bank branches.

\section{Input and output data}

The input and output data used are detailed in Table 2, where $(t)$ denotes time period and the Greek $\Delta$ denotes change in values between the start and the end of period $t$.

The inputs we have used are two: number of FTE staff and the amount of rent paid monthly. The rent is a surrogate variable used to reflect the size of the branch and its location. Other variables reflecting the potential market of the branch could be used if available because the potential of the branch to increase its outputs depends on the size of the market in which it operates, the economic status of the area, the level of competition in the area, etc (see eg Athanassopoulos $e t a l^{39}$ and Golany and Storbeck, ${ }^{40}$ who discuss the importance of including market factors in output-oriented measures). We did not have access to market size and therefore used rent as a surrogate. This relies on the assumption that a branch would attempt to negotiate the most economical rent compatible with the size and location of the branch.

The outputs we have used are detailed in Table 2. They are intended to capture the three main objectives of bank branches: (i) to increase the customer base and the sales of the branch, (ii) to serve clients that visit the branch, and (iii) to foster the use of alternative distribution channels for basic
Table 2 Inputs and Outputs used to assess operational efficiency in month $t$

\begin{tabular}{ll}
\hline Inputs & Outputs \\
\hline 1. Number Staff $(t)$ & 1. $\Delta$ Number of Clients $(t)$ \\
2. Rent $(t)$ & 2. $\Delta$ Value Current Accounts $(t)$ \\
& 3. $\Delta$ Value Other Resources $(t)$ \\
& 4. $\Delta$ Value Titles Deposited $(t)$ \\
& 5. $\Delta$ Value Credit Over Bank $(t)$ \\
& 6. $\Delta$ Value Credit Associates $(t)$ \\
& 7. Number Transactions $(t)$ \\
\hline
\end{tabular}

transactional activities. The change in the number of customers from month $t-1$ to month $t$ is intended to capture growth in clients, the change in outputs $2-6$ is intended to capture growth of various bank products, and the seventh output, number of transactions, is intended to capture the servicing activities of the bank branch. This last output is assumed to be non-discretionary. The branch needs to provide the service for general transactions but it does not wish it to increase, as transactions should be channelled to less costly media such as the Internet and the telephone.

Operational efficiency assessments should, in principle, use quantity variables rather than value variables (see eg Berger and Humphrey ${ }^{41}$ ). As quantity data were not available, we use value information concerning current accounts, other resources (which includes term deposit accounts, emigrant accounts, investment funds, savings insurance, etc), titles deposited (set of shares or bonds that the client deposits in the bank for the management of which the bank charges a certain commission), and credit. The bank under analysis distinguishes between two types of credit; directly through the bank and through associates. The former consists of all types of credit that the bank itself can provide, while the latter consists in special types of credit that the bank provides through some associate companies (like leasing or factoring credit).

The use of changes in activity levels as outputs results necessarily in some outputs being negative for some of the branches. We used the models developed in this paper to cope with such data.

\section{Results from the operational efficiency assessment}

We had monthly data on the variables in Table 2 for a set of bank branches located in the northern region of Portugal covering several months. The precise results are confidential to the bank. However, for the purposes of illustrating the RDM model, we use here data on 57 bank branches for the month of April 2001.

We used an output-oriented RDM measure, which means that the directional vector associated with inputs $\left(R_{i o}\right)$ was set to zero for all $i$. At the same time, the directional vector associated to the non-discretionary output, number of 
transactions, was also set to 0 . This is consistent with treating non-discretionary factors according to the Banker and Morey ${ }^{42}$ approach.

Results from the use of the RDM model in terms of the efficiency measures are shown in Table 3. Results in this table are sorted by the RDM ratio of norms.

The efficiency scores in Table 3 should be interpreted as distances from each bank branch to its targets (with reference to the ideal point) and therefore branches can be directly ranked and compared even when the data used to produce such results were negative.

In Table 3, we show efficiency scores for each branch as obtained directly from the RDM model, and also the ratio of norms (calculated with normalized data and based on discretionary outputs only). The difference between $1-\beta$ and the ratio of norms efficiency score relates to the existence of slacks. Therefore, the ratio of norms is never higher than the efficiency score $1-\beta$. Note that only for one bank branch (B13) there were no slacks identified in the RDM model since this model gives the same efficiency score as the ratio of norms. For all the other branches slacks were identified. The average RDM efficiency is $89.1 \%$ and the average ratio of norms efficiency is $85.44 \%$. Slacks account, therefore, on average only for a small part of inefficiency. In terms of rankings, the correlation between the rank based on the RDM efficiency score and on the ratio of norms is very high (0.9393), meaning that these efficiency scores result in very similar ranks. Nevertheless, there is a large difference in rank for some branches. For example, the highest difference happens for branch $\mathrm{B} 43$, which is ranked 19 under the RDM efficiency score and 39 when slacks are taken into account in the ratio of norms.

\section{Target setting}

Targets for each bank branch were determined using the RDM and the IRDM models. The advantage of using both the RDM and IRDM models is that we can provide alternative targets that represent different routes that a branch can choose in order to become efficient. We have chosen three inefficient units to illustrate the targets resulting from our approach. Table 4 shows the observed and target levels of units B8. B15, and B19 based on the RDM and IRDM models.

The RDM and IRDM procedures clearly give different priorities to improving different variables, with RDM targets being more demanding in certain variables, while the IRDM targets being more demanding in others. Take for example branch B19, whose output range of improvement (normalized by the maximum output so that different units of measurement do not distort our interpretations) is (1.09, $1.076,0.837,1.608,1.014,0.96$ ) (see Table 5). The highest value of this range occurs for the fourth output ( $\Delta \mathrm{Tdep}$ ), which means that the RDM measure will give priority to improving this output, while the IRDM will give priority to improving the third output ( $\Delta$ Othre), and eventually the last output ( $\Delta$ Credas). Note that the output improvements (difference between target and observed values) corresponding to the RDM and IRDM targets of branch B19, shown in Table 5, clearly indicate that factors with a higher range improve more under the RDM than the IRDM, and factors with a lower range improve more under the IRDM model. In this case, the improvements resulting from the RDM model are higher than those resulting from the IRDM model for all outputs except the third, which has the lowest range of improvement. It is, therefore, evident that the IRDM targets

Table 3 Efficiency results for April 2001

\begin{tabular}{|c|c|c|c|c|c|c|c|c|}
\hline Unit & $1-\beta$ & Ratio norm & Unit & $1-\beta$ & Ratio norm & Unit & $1-\beta$ & Ratio norm \\
\hline B1 & 1 & 1 & B32 & 0.9602 & 0.925 & B19 & 0.7971 & 0.7878 \\
\hline B10 & 1 & 1 & B25 & 0.9998 & 0.9165 & B37 & 0.8437 & 0.7859 \\
\hline B14 & 1 & 1 & B49 & 0.9282 & 0.8952 & B51 & 0.799 & 0.7778 \\
\hline B16 & 1 & 1 & B12 & 0.9061 & 0.8815 & B21 & 0.7785 & 0.7765 \\
\hline B2 & 1 & 1 & B45 & 0.9204 & 0.8748 & B50 & 0.7941 & 0.7665 \\
\hline B23 & 1 & 1 & B8 & 0.8878 & 0.8694 & B30 & 0.8671 & 0.7622 \\
\hline B28 & 1 & 1 & B36 & 0.9275 & 0.8601 & B20 & 0.8359 & 0.7608 \\
\hline B3 & 1 & 1 & B44 & 0.8673 & 0.8544 & B52 & 0.7956 & 0.76 \\
\hline B33 & 1 & 1 & B46 & 0.889 & 0.8541 & B55 & 0.7632 & 0.7542 \\
\hline B34 & 1 & 1 & B26 & 0.9225 & 0.8537 & B56 & 0.771 & 0.7388 \\
\hline B38 & 1 & 1 & B22 & 0.8836 & 0.8526 & B17 & 0.7398 & 0.7319 \\
\hline B4 & 1 & 1 & B29 & 0.9218 & 0.8485 & B60 & 0.7211 & 0.7155 \\
\hline B41 & 1 & 1 & B40 & 0.8718 & 0.8405 & B39 & 0.726 & 0.704 \\
\hline B53 & 1 & 1 & B31 & 0.9082 & 0.8404 & B48 & 0.7041 & 0.7028 \\
\hline B54 & 1 & 1 & B27 & 0.902 & 0.8276 & B18 & 0.7218 & 0.6989 \\
\hline B57 & 1 & 1 & B35 & 0.8899 & 0.8002 & B11 & 0.7274 & 0.6539 \\
\hline B58 & 1 & 1 & B15 & 0.8111 & 0.8 & B42 & 0.7451 & 0.6456 \\
\hline B7 & 1 & 1 & B13 & 0.7984 & 0.7984 & B59 & 0.7841 & 0.5655 \\
\hline B9 & 1 & 1 & B5 & 0.8388 & 0.7969 & B43 & 0.8456 & 0.421 \\
\hline
\end{tabular}


Table 4 Target levels for some units

\begin{tabular}{|c|c|c|c|c|c|c|c|c|c|}
\hline & \multicolumn{3}{|c|}{$B 8$} & \multicolumn{3}{|c|}{$B 15$} & \multicolumn{3}{|c|}{$B 19$} \\
\hline & Observed & $R D M$ & $I R D M$ & Observed & $R D M$ & $I R D M$ & Observed & $R D M$ & $I R D M$ \\
\hline Rent & 2.36 & 2.24 & 2.25 & 4.4 & 3.34 & 2.51 & 4.58 & 2.92 & 2.99 \\
\hline Staff & 4 & 4 & 4 & 7 & 5.52 & 5.73 & 5 & 5 & 5 \\
\hline$\Delta \mathrm{Cli}$ & -4 & 10.63 & 9.25 & -32 & 38.46 & 24.12 & -32 & 34.03 & 29.38 \\
\hline$\Delta$ Curac & -102.428 & -54.59 & -53.64 & -90.282 & 36.89 & 0.59 & -44.029 & 66.67 & 62.69 \\
\hline$\Delta$ Othre & 127.443 & 308.64 & 306.08 & 259.335 & 550.13 & 623.24 & 174.594 & 416.62 & 426.41 \\
\hline$\Delta \mathrm{Tdep}$ & -49.506 & -42.4 & -46.24 & -102.917 & -61.19 & -91.57 & -71.707 & -38.12 & -57.25 \\
\hline$\Delta$ Credb & 128.416 & 179.43 & 186.31 & 188.037 & 403.85 & 466.97 & -18.071 & 271.36 & 268.44 \\
\hline$\Delta$ Credas & 5.183 & 80.29 & 79.06 & 66.634 & 101.43 & 128.12 & 10.679 & 99.39 & 96.93 \\
\hline Trans & 2983 & 4027.97 & 4021.57 & 3441 & 4958.28 & 4983.33 & 3834 & 4762.5 & 4749.31 \\
\hline Rnoems & & 0.8694 & 0.8861 & & 0.8 & 0.8614 & & 0.7878 & 0.8263 \\
\hline
\end{tabular}

Table 5 Output improvements for branch B19

\begin{tabular}{|c|c|c|c|c|c|c|}
\hline & $\Delta C l i$ & $\triangle C u r a c$ & sOthre & $\Delta T d e p$ & $\triangle C r e d b$ & $\Delta$ Credas \\
\hline Range & 1.09 & 1.076 & 0.837 & 1.608 & 1.014 & 0.96 \\
\hline RDM & 66.03 & 110.99 & 242.026 & 33.59 & 289.431 & 88.711 \\
\hline IRDM & 61.38 & 106.72 & 251.82 & 14.46 & 286.511 & 86.251 \\
\hline Additive & 10.09 & 0 & 343.76 & 167.197 & 342.401 & 116.441 \\
\hline
\end{tabular}

require less effort by branch $\mathrm{B} 19$ to attain them than the RDM targets. Obviously, if branch B19 has particular difficulty in selling 'other resources' items and foresees that it cannot improve it as much as given by the IRDM targets, it has the alternative of using targets given by the RDM model.

In Table 5, we also show the improvements resulting from the additive model of Lovell and Pastor. ${ }^{14}$ These are higher than those identified by the RDM and IRDM procedures except for the case of the first two outputs, where the improvements are much lower for the additive case. One may posit that lower improvements in the first two outputs compensate for higher improvements in the remaining outputs. But arguably this is not so. In fact, using the ratio of norms to calculate the distance between branch B19 and additive targets results in a value of $67.03 \%$. This compares to 78.78 and $82.63 \%$ for the targets resulting from the RDM and IRDM model, respectively, indicating that the targets from the additive model require a higher effort from branch B19.

The targets in Table 4 may reveal additional inefficiencies apart from those associated with discretionary outputs. These should be used as informational only. For example, we can say that branch B15 could have achieved the same output targets even if it had less staff, which suggests that this bank branch is over-staffed. At the same time, all branches (B8, B15, and B19) could have achieved the same targets and also perform more transactions that seems to indicate that staff have some free time that could be used in other activities.

In terms of the whole sample, the average distances from bank branches and alternative targets can be compared through the ratio of norms. The average ratio of norms obtained for the RDM model is $85.4 \%$, the average ratio of norms obtained for the IRDM model is $88.7 \%$, and the average ratio of norms obtained for the additive model of Lovell and Pastor ${ }^{14}$ is $81.59 \%$. These values show that on an average the IRDM model provides closer targets to production units, followed by the RDM model, and finally by the additive model that tends to provide the furthest targets as expressed through the lowest average ratio of norms.

As usual in DEA analyses apart from targets, we can also provide inefficient units with peer or reference units to which they can compare themselves. For the case of branch B19, Table 6 presents the reference units as given by the RDM and IRDM models. The peers presented can be used by branch B19 as benchmarks of performance. All of these benchmarks perform better than branch B19 in most respects, while having less favourable conditions in terms of rent (location and space) and transactions (see also transactions per staff in Table 6). Note that branch B58 dominates the peer set both for the RDM and IRDM measures. This means that this branch should be looked at by branch B19 as the main benchmark unit whatever the direction it chooses to follow towards the efficient frontier.

The above analysis is especially meaningful when undertaken over time so that consistent efficient or inefficient behaviour is identified in bank branches. For managerial purposes, it is interesting to analyse efficiency and productivity change over time. These changes could be analysed in more detail through Malmquist index approaches adapted to the present RDM measure. These issues are currently the subject of further research.

It can be shown (though we do not pursue it in this paper) that the use of unrestricted in sign differences as output 
Table 6 Peers of branch B19

\begin{tabular}{|c|c|c|c|c|c|c|}
\hline & $\begin{array}{c}R D M \\
I R D M \\
B 19\end{array}$ & $\begin{array}{c}0.448 \\
0.4459 \\
B 58\end{array}$ & $\begin{array}{c}0.2374 \\
0.2315 \\
B 38\end{array}$ & $\begin{array}{c}0.1573 \\
0.1425 \\
B 28\end{array}$ & $\begin{array}{c}0.1573 \\
0.1425 \\
B 9\end{array}$ & $\begin{array}{c}0 \\
0.0375 \\
B 10\end{array}$ \\
\hline Rent & 4.58 & 3.352 & 3.093 & 1.55 & 2.82 & 4.1 \\
\hline Staff & 5 & 5 & 5 & 3 & 7 & 5 \\
\hline$\Delta \mathrm{Cli}$ & -32 & -21 & -29 & -21 & 341 & -4 \\
\hline$\Delta$ Curac & -44.029 & -124.5 & 582.99 & -11.891 & -87.798 & -68.575 \\
\hline$\Delta$ Othre & 174.594 & 598.782 & -108.349 & 36.482 & 1070.42 & 712.623 \\
\hline$\Delta$ Tdep & -71.707 & 118.011 & -80.002 & -76.75 & -381.042 & -695.793 \\
\hline$\Delta$ Credb & -18.071 & 338.803 & 211.575 & 57.072 & 383.77 & 147.824 \\
\hline$\Delta$ Credas & 10.679 & 130.86 & 97.964 & 57.352 & 53.91 & 1.122 \\
\hline Trans & 3834 & 3846 & 5322 & 3567.14 & 7724 & 5142 \\
\hline Trans/staff & 766.8 & 769.2 & 1064.4 & 1189.047 & 1103.43 & 1028.4 \\
\hline
\end{tabular}

variables, as we do here, is equivalent to using the original non-negative variables from which the differences are derived (with values at the beginning of a time period on the input side and values at the end of that period on the output side), with certain constraints on their DEA weights. In our case, this option would result in six more inputs, while changing outputs from changes to actual values at the end of April. Targets would be less easy to interpret because they would imply trade-offs in outputs, but also in inputs due to our weights restrictions. The use of the RDM model is, therefore, preferable because it requires less factors to be used in the analysis and it facilitates the interpretation of the resulting targets.

\section{Conclusion}

In the presence of negative data, traditional radial models for efficiency assessment cannot be used without transforming the data, as they move negative inputs/outputs in the wrong direction. The standard additive model is the main efficiency assessment tool that has been used in these cases, because of its translation-invariant properties (and units invariant in some cases). The additive model has, however, two main disadvantages that are overcome in this paper: it tends to project units on the furthest points of the frontier, therefore implying unnecessary efforts by production units, and it does not provide a final efficiency measure by which comparisons and rankings can he made.

In this paper, we developed a model based on the directional distance function approach, where the direction is the range of possible improvement (defined as maximum output minus observed output, or observed input minus minimum input). We call this model RDM. The RDM model is units and translation invariant, which makes it suitable to be used in the presence of negative data. In addition, the RDM model results in an efficiency measure that is very similar to those used in radial models except that the point with reference to which efficiency is measured is no longer the origin but an ideal point (having maximum outputs and minimum inputs). Such a measure represents an interesting development in the literature as there was to date no radial or non-radial efficiency measure, to the authors' knowledge, that could be applied directly to negative data.

We extended our approach by considering a variant of the RDM model, where the directional vector is the inverse of the range of possible improvement. The resulting model (IRDM) has the advantage of prioritizing improvement of the factors on which the unit performs best, and therefore it tends to yield closer targets to the assessed unit than the RDM model or the well-known additive model. The RDM and IRDM were applied in this paper to a sample of Portuguese bank branches. The advantage of using both specifications is that bank branches can choose from different types of targets (one prioritizing improvements on the factors on which the unit performs worst, and the other prioritizing improvement of the factors on which the unit performs best) both leading to the production frontier.

Acknowledgements-We acknowledge the financial support of the Portuguese Foundation for Science and Technology, and the European Social Fund. The contents of the paper are the responsibility of the authors.

\section{References}

1 Farrell MJ (1957). The measurement of productive efficiency. $J$ R Stat Soc Ser A 120: 253-281.

2 Charnes A, Cooper WW and Rhodes E (1978). Measuring efficiency of decision making units. Eur J Opl Res 2: 429-444.

3 Drake L and Howcroft B (1995). Measuring the relative efficiency of the selling function: an application of data envelopment analysis to UK bank branches. Working Paper No. 89/95, Loughborough University Banking Centre, UK.

4 Howcroft JB and Beckett A (1993). Change in the UK bank branch networks: a customer perspective. Service Ind $J$ 13: $267-288$. 
5 Howcroft JB (1992). Contemporary issues in UK bank delivery systems. Int $J$ Service Ind M 3(1): 39-56.

6 Howland A (2000). The evolution of the bank branch. Canadian Banker 6: 25-29.

7 Cook WD, Hababou M and Tuenter HJH (2000). Multicomponent efficiency measurement and shared inputs in data envelopment analysis: an application to sales and service performance in bank branches. J Prod Anal 14: 209-224.

8 Cook WD and Hababou M (2001). Sales performance measurement in bank branches. Omega Int J Mngt Sci 29: 299-307.

9 Pastor JT (1994). How to discount environmental effects in DEA: an application to bank branches. Working Paper No. 011/94, Depto. De Estadistica e Investigacion Operativa, Universidad de Alicante, Spain.

10 Lovell CAK (1995). Measuring the macroeconomic performance of the Taiwanese economy. Int J Prod Econ 39: 165-178.

11 Seiford ML and Zhu J (2002). Modeling undesirable factors in efficiency evaluation. Eur $J$ Opl Res 142: 16-20.

12 Charnes A et al (1985). Foundations of data envelopment analysis for Pareto-Koopmans efficient empirical production functions. $J$ Econ 30: 91-107.

13 Ali AI and Seiford LM (1990). Translation invariance in data envelopment analysis. Opns Res Lett 9: 403-405.

14 Lovell CAK and Pastor JT (1995). Units invariant and translation invariant DEA models. Opns Res Lett 18: 147-151.

15 Pastor JT (1996). Translation invariance in data envelopment analysis: a generalisation. Ann Opns Res 66: 93-102.

16 Thrall RM (1996). The lack of invariance of optimal dual solutions under translation. Ann Opns Res 66: 103-108.

17 Banker RD, Charnes A and Cooper WW (1984). Source models for estimating technical and scale inefficiencies in data envelopment analysis. Mngt Sci 30: 1078-1092.

18 Chambers RG, Chung Y and Färe R (1996). Benefit and distance functions. $J$ Econ Theory 70: 407-419.

19 Chambers RG, Chung Y and Färe R (1998). Profit, directional distance functions, and Nerlovian efficiency. J Optim Theory Appl 98: 351-364.

20 Coelli T (1998). A multi-stage methodology for the solution of orientated DEA models. Opns Res Lett 23: 143-149.

21 Frei FX and Harker PT (1999). Projections onto efficient frontiers: theoretical and computational extensions to DEA. J Prod Anal 11: 275-300.

22 Cherchye L and Van Puyenbroeck T (2001). A comment on multi-stage DEA methodology. Opns Res Lett 28: 93-98.

23 Portela MCS, Borges P and Thanassoulis E (2003). Finding closest targets in non-oriented DEA models: the case of convex and non-convex technologies. J Prod Anal 19(2/3): 251-269.

24 Krivonozhko VE, Utkin OB, Volodin AV and Sablin IA (2001). Application of DEA approach to production units with some negative outputs. Paper Presented at OR43, University of Bath, UK, 4-6 September.

25 Färe R, Grosskopf S and Lovell AK (1994). Production Frontiers. Cambridge University Press: Cambridge, UK.

26 Chen Y and Ali AI (2002). Output-input ratio analysis and DEA frontier. Eur J Opl Res 142: 476-479.
27 Allen K (1999). Dea in the ecological context - an overview. In: Westermann G (ed). Data Envelopment Analysis in the Service Sector. Gabler Edition Wissenschaft: Harzer, pp 203-235.

28 Dyckhoff $\mathrm{H}$ and Allen K (2001). Measuring ecological efficiency with data envelopment analysis. Eur J Opl Res 132: 312-325.

29 Chung Y, Färe R and Grosskopf S (1997). Productivity and undesirable outputs: a directional distance function approach. J Environ Mngt 51: 229-240.

30 Thanassoulis E (2001). Introduction to the Theory and Application of Data Envelopment Analysis: A Foundation Text with Intefrated Software. Kluwer Academic Publishers: Dordrecht.

31 Cooper WW, Park KS and Pastor JT (1999). RAM: a range measure of inefficiency for use with additive models, and relations to other models and measures in DEA. J Prod Anal 11: 5-42.

32 Bogetoft P and Hougaard JL (1998). Efficiency evaluations based on potential (non-proportional) improvements. J Prod Anal 12: 233-247.

33 Asmild M, Hougaard JL, Kronborg D and Kvist HK (2003). Measuring inefficiency via potential improvements. J Prod Anal 19: $59-76$.

34 Ali AI and Seiford LM (1993). The mathematical programming approach to efficiency analysis. In: Fried HO, Knox Lovell CA and Schmidt SS (eds). The Measurement of Production Efficiency: Techniques and Applications. Oxford University Press, New York, Oxford, pp 120-159.

35 Cherchye L and Van Puyenbroeck T (1999). Learning from input-output mixes in DEA: a proportional measure for slackbased efficient projections. Managerial Decis Econ 20: 151-161.

36 Cherchye L and Van Puyenbroeck T (1999). Non-radial efficiency and semi-radial efficiency. In: Westermann $G$ (ed). Data envelopment Analysis in the Service Sector. Gabler Edition Wissenschaft: Harzer, pp 51-64.

37 Charnes A, Haag S, Jaska P and Semple J (1992). Sensitivity of efficiency classifications in the additive model of data envelopment analysis. Int $J$ Systems Sci 23: 789-798.

38 Briec W (1998). Hölder distance function and measurement of technical efficiency. J Prod Anal 11: 111-131.

39 Athanassopoulos AD, Soteriou AC and Zenios SA (2000). Disentangling within- and between-country efficiency differences of bank branches. In: Harker PT and Zenios SA (eds). Performance of Financial Institutions; Efficiency. Innovation and Regulation. Cambridge University Press, Cambridge, UK, pp 336-363.

40 Golany B and Storbeck JE (1999). A data envelopment analysis of the operational efficiency of bank branches. Interfaces 29(3): $14-26$.

41 Berger AN and Humphrey DB (1997). Efficiency of financial institutions: international survey and directions for future research. Eur J Opl Res 98: 175-212.

42 Banker RD and Morey RC (1986). Efficiency analysis for exogenously fixed inputs and outputs. Opns Res 34: 513-520. 